\title{
Novel Approaches in Microstructure Evaluation of Polymer Blends
}

\author{
Nisha Preschilla ${ }^{*}$, Nitin Moghe ${ }^{*}$, Orrie Riccobono ${ }^{* *}$, Sandeep Tyagi ${ }^{*}$, Srinivasan Rajagopalan ${ }^{*}$ \\ *GE India Technology Center, Hoodi Village, Whitefield Road, Bangalore-560066, India \\ ${ }^{* *}$ GE Advanced Materials, One Noryl Avenue, Silkirk, NY-12158, USA
}

The increased replacement of metals and ceramics by polymeric materials in automotive, consumer goods and medical devices has resulted in a demand for faster methods to study the microstructure of these materials. Traditionally, microstructural analysis of polymeric materials has been carried out with Transmission Electron Microscopy (TEM). However, specimen preparation for TEM analysis is tedious and skill-intensive and TEM is not generally a viable option for polymeric materials containing inorganic fillers due to ultramicrotomy limitations. This report highlights some of the alternative methods we have employed based on preferential etching of phases in polymer blends by exposure to plasma or solvents [1] to generate the necessary topographical contrast, which can then be imaged using Scanning Electron Microscopy (SEM). These techniques have greatly simplified specimen preparation and have resulted in faster analysis and increased productivity. A few examples depicting these alternative methods, including cases where they have also proven to be superior techniques to TEM, are discussed below.

For fiber-reinforced polymer blends, comprehensive information required on network morphology and fiber diameter distribution that affects overall performance of the blend is conventionally obtained through detailed fracture surface SEM analysis. However, in the case of a fibrous polymeric second phase (e.g. polyethylene, polyethylene terephthalate etc.) dispersed in a matrix polymer, fracture surface analysis may not illustrate the true network morphology since fracturing of the sample itself can alter the fiber dispersion and dimensions. The observed network morphology from fracture surface analysis may therefore provide an imprecise picture. Etching the matrix phase at a highly controlled rate by exposure to plasma followed by SEM imaging to study fiber dispersion, has proven to be an attractive replacement of time-consuming, and occasionally inaccurate, fracture surface analysis. Figure 1 illustrates the true fiber network recorded following etching of the matrix phase with oxygen plasma (Figure 1a) and comparison with the fracture surface SEM (Figure 1b); demonstrating the deficiency in traditional analysis technique in replicating the isotropic network that the fiber takes within the polymer. Figure $2 \mathrm{a}$ provides another example wherein oxygen plasma etch-SEM has been successfully employed to analyze the microstructure of a polymer blend containing polycarbonate (PC), styrene acrylonitrile (SAN), and a rubbery impact modifier also containing various common inorganic additives. Because of differential etching rates of these components, plasma etching readily provides information on the microstructural features in great detail. A comparison with TEM micrographs (Figure 2b) shows good correlation between the plasma etching method and traditional TEM analysis. Note that for TEM analysis, the presence of inorganic additives that restrict ultramicrotomy, along with complex staining processes, stand as major disadvantages.

Dissolution of selected phases in a polymer blend with suitable solvents is another etching method for morphology evaluation of polymer blends. Figure 3a illustrates this technique with a SEM micrograph showing dispersion of polyphenylene ether (PPE) into a polyamide (PA) matrix, wherein the PPE phase has been extracted with toluene. This analytical technique proves to be an 
efficient tool in monitoring PPE dispersion and size distribution (especially on the surface), since these factors are believed to play a role in controlling physical properties and paintability. A comparison with TEM (Figure $3 \mathrm{~b}$ ) demonstrates that though similar information is obtained, a slight compression of rubbers is induced during microtomy. This compression is unavoidable, even under cryo conditions. As previously mentioned, TEM analysis is impracticable for formulations with high inorganic filler concentration. The etching-SEM technique has additional advantages over TEM and SPM for studying in-plane PPE dispersion on the surface (Figure 3c), providing faster and more accurate information with less bias from sample preparation.

\section{Reference}

1. L.C. Sawyer and D.T. Grubb, Polymer Microscopy, Chapman \& Hill, 1996.
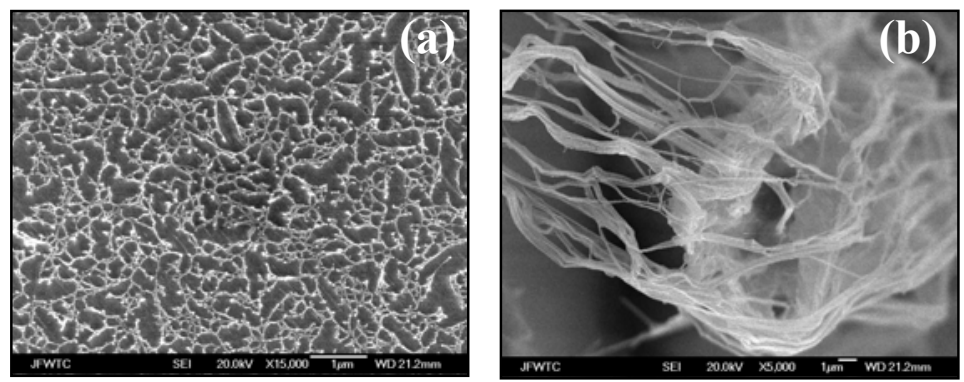

Figure 1. SEM micrographs showing the dispersion of a polymeric fiber phase studied through (a) plasmaetching and (b) fracture surface analysis.
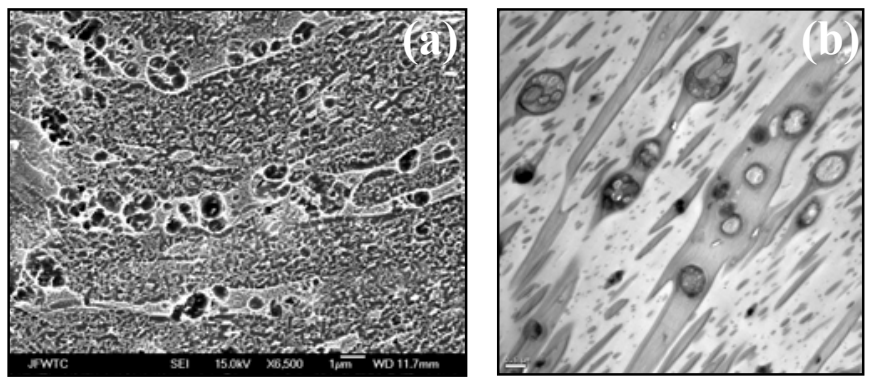

Figure 2. (a) SEM micrograph of a plasma etched blend of PC, SAN and rubbery impact modifier blend and (b) comparison to TEM. Dispersed particles seen in the micrographs are the rubbery impact modifiers.
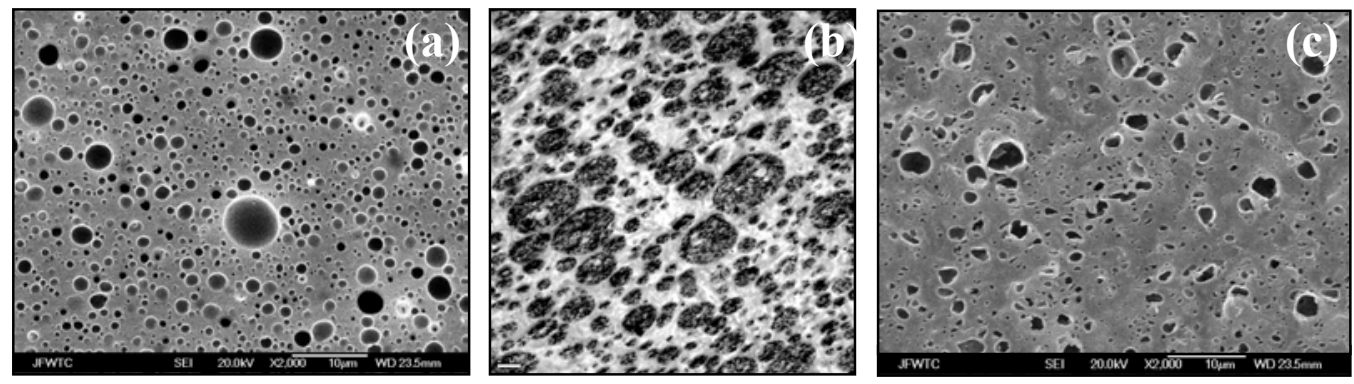

Figure 3. (a) Toluene etched SEM micrograph of a PPE / PA blend, showing dispersion of PPE in PA and (b) TEM of PPE / PA blend. It can be see that SEM analysis provides a more accurate morphology; evading the slight compression of domains, which is a sample preparation induced artifact. PPE dispersion on the surface of the PPE / PA blend is shown in (c). 